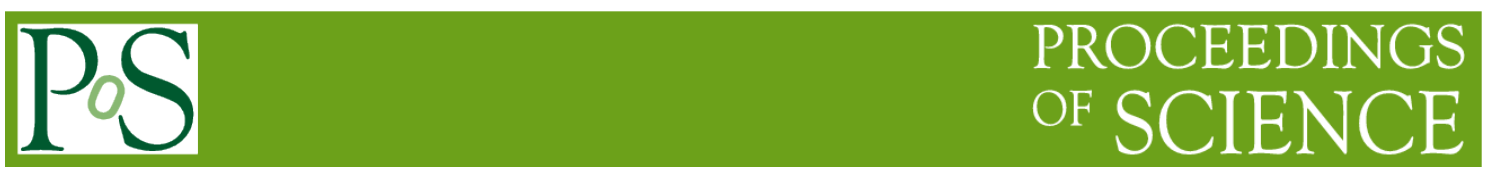

\title{
Solar Proton Transport to Earth
}

\author{
Edward Bramlitt ${ }^{1}$ \\ Bramlitt Consulting Services \\ 8813 Camino Osito NE, Albuquerque, NM 87111 USA \\ E-mail: ebramlittemsn.com
}

\begin{abstract}
Since 1976 the USA weather satellites named GOES, followed by a sequence number, have continuously monitored the Sun for proton emissions. The monitoring data is a valuable resource available online, but it is not being fully utilized. To encourage use, procedures are detailed for downloading GOES data to a Windows 10 computer with Excel software. Excel makes easy the calculation of proton velocity, pace, and rigidity given energy, which GOES registers in eleven channels from 2.5 to $>700 \mathrm{MeV}$. Plots of pace versus time protons arrive at GOES are linear, zero pace is emission time, and line slope is proton path length. Proton flux at GOES is an indicator of neutron flux at commercial aircraft on polar routes, and dose to aircraft occupants is calculable from flux. January 2014 is chosen to demonstrate some uses of GOES data. Proton emission started on January 6 around 7:30, flux of $100 \mathrm{MeV}$ protons began a sharp increase coincident with neutron monitor count rate increases causing GLE 72, and the emission continued for four days with several emission bursts. The January 8 burst ( 25 -hr width at half max) is shown to cause $>6$ milli-Sievert dose at highlatitude flight levels. For perspective, Germany requires air crewmembers be grounded by that dose and have medical follow-up. In conclusion, GOES data cover 270 proton events during which there were 41 GLE, so January 2014 is a tip of the iceberg.
\end{abstract}

35th International Cosmic Ray Conference - ICRC2017

10-20 July, 2017

Bexco, Busan, Korea

${ }^{1}$ Speaker 


\section{Introduction}

Satellites named GOES, followed by a sequence number, are a resource of the USA National Oceanic and Atmospheric Administration (NOAA) for monitoring space and terrestrial weather. Only GOES 13 and 15 are currently operational, but GOES have continuously monitored the Sun for proton emissions since 1976. The NOAA Space Weather Prediction Center (SWPC) evaluates GOES data and reports a solar proton event (SPE) when proton flux is significant. From 1976 to 2017 there were 270 SPE that caused 35 ground level enhancements (GLE). (Six more GLE had no reported association with SPE.)

GOES registers protons in 11 energy channels $\mathrm{P} 1$ at $2.5 \mathrm{MeV}$ to $\mathrm{P} 11$ at $>700 \mathrm{MeV}$. The data is a valuable resource for solar physics studies and air travel radiation safety, but it has not been fully utilized or understood. For example, Thakur et al. [1] say a P11 flux increase is typical of GLE, it increased $67 \%$ at GOES 13 on 6 January 2014 confirming the observation of GLE 72 at neutron monitor (NM) station SOPO, and flux was greater at GOES 13 than 15 at high energies.

GOES data say otherwise. The GLE started around 8:00, and from 7:15 to 9:15 the P11 flux did not exceed background plus $2 \sigma$ at GOES 15 and only from 8:30 to 8:45 at GOES 13 . Flux decreased with channel number from the maximum at P1 to P11 which was 200,000 times smaller. The GOES 15/GOES 13 fluxes averaged $1.06 \pm 0.17$ for all channels other than P2. But, $100 \mathrm{MeV}$ proton fluxes at GOES 13 and 15 began increasing at the GLE start time, and that GOES data confirms the GLE 72 on 6 January 2014.

Polar-route flights have become common since starting in 2001. The USA Federal Aviation Administration (FAA) defines them as having any time beyond $78^{\circ} \mathrm{N}$ or $60^{\circ} \mathrm{S}$, and it requires airlines flying those routes to mitigate dose during solar flare activity [2]. It reports estimated dose from galactic cosmic rays (GCR) for representative domestic and international flights [3], but not from SPE, and it has not changed the representatives since the first in 1990.

SPE proton create neutrons in air, and $72 \mathrm{kPa}$ of air at NM SOPO did not shield the NM from neutrons that caused GLE 72. Commercial planes cruise in air at $20 \mathrm{kPa}$, so some polar-route flights received a neutron dose on 6 January 2014.

Section 2 makes using GOES data easy by telling how to download it to a personal computer, Section 3 has characteristics of the solar protons transported to GOES, Section 4 demonstrates a GOES data use, and Section 5 concludes GOES data is valuable for solar physics, air travel radiation safety, and the understanding of GLE.

\section{GOES Data}

Space weather by GOES is at www.swpc.noaa.gov with charts of solar proton flux and more, but chart data is at http://satdat.ngdc.noaa.gov. Get data by selecting GOES SEM Data Files $\rightarrow$ new-avg, pick a year, month, GOES number, and csv to display data files. Click a file name with epead for channels 1-7 or hepad for channels 8-11; e.g., epead_p17ew_5m or hepad_ap_5m. A file with $1 m$ or $5 m$ has data at 1-min or 5-min intervals, ap has alpha and proton flux, and xrs has $\mathrm{X}$ ray flux. Clicked files go to the computer download directory. 
To work a download, open a new Excel file, select Data $\rightarrow$ Get External Data $\rightarrow$ From Text. Find downloads, highlight a GOES file there, click Import $\rightarrow$ Delimited $\rightarrow$ Next $\rightarrow$ Comma $\rightarrow$ Next $\rightarrow$ Finish, and Put Data in Existing Worksheet $\rightarrow$ OK. Create a folder named GOES Data and save the file to it using the file name in cell A1, but for easy retrieval, name it by year, month, GOES number, data type, and count time. For example, $201401 \mathrm{~g} 15$ hepad 5 min.

The first few hundred rows of column A have important descriptive information, and below them is the header time-tag for 288 or 1,440 rows at each day in the month. Data headers are on the timetag row typically with four columns for each proton channel. Copy header row and rows for days of interest to a new sheet. Find any rows with -9999 for missing data and remove the -9999 s. Insert scatter chart of time and "corrected flux" columns for an overview. Copy columns of interest with time-tag and headers to new sheets for analysis using more scatter charts.

\section{Solar Protons at GOES}

Energy (E) of an atomic particle is related to its mass $(\mathrm{m})$ by the expression $E=(\gamma-1) \mathrm{mc}^{2}$ where $\gamma$ is the Lorentz factor and $\mathrm{c}$ is light speed. Table 1 is an Excel sheet with solutions to the expression in cells E7- H17 for the characteristics of protons with E of the GOES channels. To help others, A7-A14 have text versions of formulas in E7-H7 that use constants in B1-B3.

\begin{tabular}{|c|c|c|c|c|c|c|c|c|}
\hline 4 & A & B & C & D & $E$ & $\mathbf{F}$ & G & $\mathrm{H}$ \\
\hline 1 & Light Speed & $299,792.46$ & $\mathrm{~km} \mathrm{~s}^{-1}$ & & & & & \\
\hline 2 & Astrnomical Unit & $149,597,870.70$ & $\mathrm{~km}$ & & & & & \\
\hline 3 & Proton Mass & 938 & $\mathrm{MeV}$ & & & & & \\
\hline \multicolumn{9}{|l|}{4} \\
\hline 5 & & & Channel & Energy & Velocity & Pace & Rigidity & Lorentz \\
\hline 6 & & & & (MeV) & $(\mathrm{km} / \mathrm{s})$ & (min/AU) & (GV) & \\
\hline 7 & Velocity & & P1 & 2.5 & 21,844 & 114 & 0.07 & 1.003 \\
\hline 8 & \multicolumn{2}{|c|}{$=S Q R T\left(\left(1-(\$ B \$ 3 /(\$ B \$ 3+D 7))^{\wedge} 2\right)\right)^{*} \$ B \$ 1$} & P2 & 6.5 & 35,111 & 71.0 & 0.11 & 1.007 \\
\hline 9 & Pace & & P3 & 11.6 & 46,716 & 53.4 & 0.15 & 1.012 \\
\hline 10 & $=\$ B \$ 2 / E 7 / 60$ & & P4 & 30.6 & 74,760 & 33.4 & 0.24 & 1.033 \\
\hline 11 & Rigidity & & P5 & 63.1 & 104,751 & 23.8 & 0.35 & 1.067 \\
\hline 12 & \multicolumn{2}{|c|}{$=S Q R T\left(H 7^{\wedge} 2-1\right)^{*} \$ B \$ 3 / 1000$} & P6 & 165 & 157,728 & 15.8 & 0.58 & 1.18 \\
\hline 13 & Lorentz & & P7 & 433 & 218,645 & 11.4 & 1.00 & 1.46 \\
\hline 14 & \multicolumn{2}{|c|}{$=1 / S Q R T\left(1-E 7^{\wedge} 2 / \$ B \$ 1^{\wedge} 2\right)$} & P8 & 375 & 209,778 & 11.9 & 0.92 & 1.40 \\
\hline 15 & & & P9 & 465 & 222,941 & 11.2 & 1.04 & 1.50 \\
\hline 16 & & & P10 & 605 & 238,038 & 10.5 & 1.23 & 1.64 \\
\hline 17 & & & P11 & 700 & 245,770 & 10.1 & 1.34 & 1.75 \\
\hline
\end{tabular}

Table 1. Excel sheet with characteristics of solar protons transported to GOES channels P1P11. A7-A14 have text versions of formulas at E7-H7 that use constants at B1-B3 to calculate the characteristics at E7-H17..

The $\mathrm{E}$ in Table 1 are from early calibrations made at ground level that gave channels with varying widths plus a representative $\mathrm{E}$ for each channel. Channels P1-P7 on operational GOES were calibrated recently relative to proton detectors on other satellites [4]. The calibrations are named here as Eg for ground and Es for space. The Es P1-P7 values are 2.5, 6.0, 11.6, 24.5, 56.6,115, and $287 \mathrm{MeV}$. NOAA has not endorsed the Es. 


\section{Using GOES Data}

January 2014 is picked to demonstrate GOES data use. Figure 1 plots NM SOPO count rates and GOES 13 and 15 channels P5 and P6 fluxes on January 6. Only P5 and P6 had flux increases near the GLE start. All data are at 5-min intervals, and backgrounds were made the average rate or flux from 2:00 to 6:00. The NM count rate rose above background plus $2 \sigma$ at 8:10 and remained continuously above until 10:35. Significant flux increases began for GOES 13 and 15 P5 at 8:10 and 8:15, respectively, and P6 at 8:05. GOES data support the reality of GLE 72 and with an 8:10 start time.

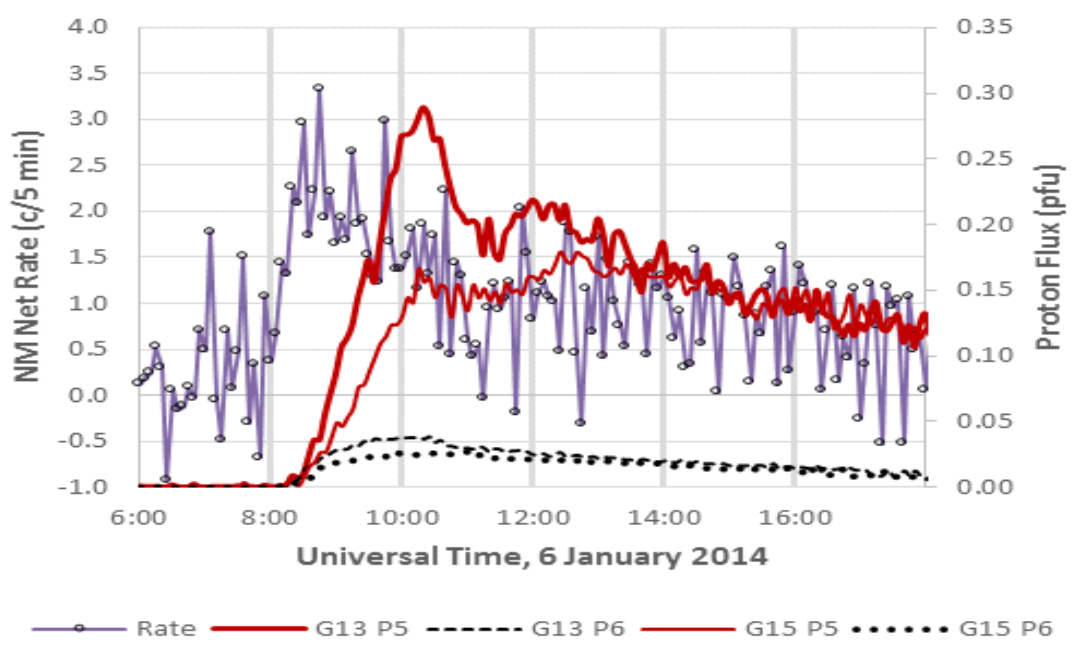

Figure 1. Count rates at NM station SOPO and proton fluxes at GOES 13 and 15. The NM and GOES 13 P5 registered significant increases at 8:10; at 8:15 for GOES 15 P5 and 8:05 for both GOES P6. GLE 72 is real and with an 8:10 start by 5 -min data.

The Eg and Es for channel P5 is 63.1 and $56.6 \mathrm{MeV}$; for P6 it is 165 and $115 \mathrm{MeV}$. The range is 56.6 to $165 \mathrm{MeV}$, so " $100 \mathrm{MeV}$ " is adopted to represent the solar protons of Figure 1. There is $<1 \mathrm{sec}$ time difference between the arrival of protons at GOES and neutrons $>10 \mathrm{MeV}$ at NM. By flux and counts at 5-min intervals, GLE 72 started at 8:10 due to neutrons created by $100 \mathrm{MeV}$ solar protons.

SWPC reported a GOES detection of SPE protons on 6 January 2014 when the sum of fluxes from all channels $>10 \mathrm{MeV}$ exceeded $10 \mathrm{pfu}$. (A pfu is proton flux unit and is $1 \mathrm{proton} / \mathrm{cm}^{2} / \mathrm{s}$.) The flux increase started at 9:15, reached a maximum of $42 \mathrm{pfu}$ at 16:00, decreased but remained above $10 \mathrm{pfu}$, and rose to a second maximum of 1,033 pfu at 3:40 on January 9 . SWPC does not report SPE durations but says they end when the integral flux drops below 10 pfu. Time from start to first and second maxima were 6.8 and 66.3 hours.

The GOES differential energy data tell a different story. Each proton channel had one or more flux peaks of different start time and magnitude, and they indicate two overlapping SPE with different start and end times. Plots of flux vs. time give peak maximum flux, full width at half maximum (FWHM), and time of maximum. SPE 1 duration from its start to the start of SPE 2 was 36 hours, and SPE 2 lasted more than 50 hours . 
Table 2 has characteristics of SPE 1 to the left and SPE 2 to the right. Channels P8-P11 are excluded as their fluxes were too small to yield meaningful measures. The SPE 1 and 2 average FWHM are 10 and 16 hours; their total fluxes are 57 and 2,258 pfu. SPE 2 was 40 times more intense based on flux.

\begin{tabular}{|c|c|c|c|c|c|c|}
\hline $\begin{array}{c}\text { Channel } \\
(\mathrm{P} \#)\end{array}$ & $\begin{array}{c}\text { FWHM } \\
(\mathrm{hr})\end{array}$ & $\begin{array}{c}\text { Peak Time } \\
(\mathrm{m} / \mathrm{d} / \mathrm{y} \text { h:m })\end{array}$ & $\begin{array}{c}\text { Flux } \\
(\mathrm{pfu})\end{array}$ & $\begin{array}{c}\text { FWHM } \\
(\mathrm{hr})\end{array}$ & $\begin{array}{c}\text { Peak Time } \\
(\mathrm{m} / \mathrm{d} / \mathrm{y} / \mathrm{h}: \mathrm{m})\end{array}$ & $\begin{array}{c}\text { Flux } \\
(\mathrm{pfu})\end{array}$ \\
\hline P1 & 12 & $18681020: 00$ & 50 & 22 & $1 / 10 / 140: 05$ & 1600 \\
\hline P2 & 11 & $1 / 6 / 1416: 00$ & 3 & 10 & $1 / 9 / 147: 00$ & 500 \\
\hline P3 & 8 & $1 / 6 / 1415: 00$ & 2.5 & 25 & $1 / 8 / 1421: 15$ & 120 \\
\hline P4 & 18 & $1 / 6 / 1419: 00$ & 1.2 & 26 & $1 / 8 / 1416: 00$ & 35 \\
\hline P5 & 12 & $1 / 6 / 1415: 20$ & 0.18 & 8 & $1 / 8 / 143: 00$ & 2.5 \\
\hline P6 & 8.6 & $1 / 6 / 1413: 00$ & 0.028 & 11 & $1 / 8 / 142: 25$ & 0.045 \\
\hline P7 & 4.4 & $1 / 6 / 1411: 00$ & 0.009 & 8.3 & $1 / 8 / 141: 15$ & 0.01 \\
\hline \hline
\end{tabular}

Table 2. Features of proton flux peaks at GOES energy channels P1 to P7 during SPE in January 2014. SPE 1 are columns 2-4 and SPE 2 are columns 5-7.

Charts were made of pace vs. time-of-day when SPE 1 protons first reached GOES 13 and 15, and separately for Eg and Es. Channels P1 and P11 were not included as P1 had too many peaks and P11 had none. The charts say solar emission time was 7:33 (Eg) and 7:23 (Es) by GOES 13 and 7:36 (Eg) and 7:27 (Es) by GOES 15. The average is 7:30 $\pm 5.2 \mathrm{~min}$. Path lengths were 1.40, 1.47, 2.25, and 2.34 AU. Similar charts were made for SPE 2 and GOES 15 channels P1-P7. Emission started January 7 at 18:25 (Eg) or 18:20 (Es) with path lengths 3.73 or 3.80 AU. SPE 1 ended on January 7 around 19:25 to 20:15 when SPE 2 began.

SPE proton emission times are important for solar physics, but other SPE features like energy, flux, and duration are of concern to air travel radiation safety. The potential for highest doses lies with long-lived high flux SPE and long-haul high-latitude flights such as New YorkShanghai and New York-Beijing. One USA airline operates those flights daily in both directions with flight times around 13 to 15 hours.

Dose estimates can be the product of exposure time, flux, and a fluence-to-dose coefficient. Exposure could be 10 hours on 8 January 2014 as flight times and flux FWHM are much longer, but some of the flight time is at lower latitudes and flight levels. A 5-hr exposure is adopted here for dose estimates, and those hours are from 11:00 to 16:00.

Dose estimates normally employ coefficients for an anterior-to-posterior (A-P) exposure geometry [5], but an isotropic geometry is recommended with GCR at flight levels [6]. By digitizing plots in those references, neutron dose coefficients with $\mathrm{pSv} \mathrm{cm}^{2}$ units are $470 \pm 3 \%$ (A-P) and $345 \pm 6 \%$ (isotropic) from 10 to $165 \mathrm{MeV}$. (The relative errors are by the mean of 13 samples and $1 \sigma$ uncertainty.) Arguments can be made for and against both coefficients, so both coefficients are used and two estimates are given.

Neutron flux at polar flight levels derives from solar proton flux above the polar atmosphere. GOES channels P3-P6 (10 to $165 \mathrm{MeV}$ ) are chosen to represent the proton flux. Other channels are neglected because of low energy or low flux. No evidence is given for the polar flux to equal the P3-P6 flux, and it would be larger if GOES registers protons of rigidity $>0 \mathrm{GV}$. As a single dose coefficient applies for the energy range, fluxes at channels P3-P6 are summed without weights. The neutron flux must be less than proton flux, and it is set at 20\% of the P3-P6 sum for flight levels. No evidence is given for this percentage, but, neutrons from SPE 1 caused GLE 72, and proton flux was 40 times greater with SPE 2. 
The two dose estimates are 10 and $7.3 \mathrm{mS}$ for high latitude commercial flights on 8 January 2014, using P3-P6 fluxes averaged for GOES 13 and 15 and other factors stated above. Significantly, the main dose contributor was $\mathrm{P} 3$, then $\mathrm{P} 4$, and so on as that is the order of decreasing flux, and the dose coefficients were constant over the P3-P6 energy range. But, dose coefficients rise sharply with further energy increases, so the contribution from channels $>$ P6 may be significant with other SPE. Surprisingly, dose was not due to $\mathrm{GeV}$ protons.

\section{Conclusion}

Protons have been transported from the Sun, on 270 occasions since 1976, to the GOES satellites where their energy and flux are measured, or they proceed into the atmosphere and create neutrons that cause radiation dose at flight levels or GLE at NM. The proton data accumulated at GOES is online and easily downloaded to a personal computer with spreadsheet software. GOES X ray data can be included to associate with the proton data. Proton pace is a parameter first used here to determine solar proton emission time and proton path length. GOES and NM data were combined to confirm the reality of a very weak GLE, and more importantly, specific proton energy channels were associated with NM count history. There are 35 SPE-GLE connections, and the approach could be applied with them to learn more about GLE. The example GOES data use had two SPE, and by creating multiple spreadsheet scatter charts for all GOES channels, new SPE features emerge, including arrival times, flux peaks, FWHM, maximum fluxes, and SPE durations. One of the two SPE was intense throughout January 8 and may have caused dose $>6 \mathrm{mSv}$ to aircraft on long-haul high latitude flights. GOES data could be used to reconstruct doses for many of the SPE, but a key factor that needs resolution is neutron flux at flight levels and NM relative to proton flux at GOES. Ultimately, SWPC might generate dose estimates as SPE are occurring for the benefit of air travelers.

Acknowledgement. This unfunded work benefited by using data from the NMDB database at www.nmdb.eu which was founded under the European Union's FP7 program (contract no. 213007), and special thanks to the University of Delaware for providing NM SOPO data to the NMDB as part of receiving support from the USA National Science Foundation under grant ANT-0838839.

\section{References}

[1] N. Thakur, N. Gopalswamy, H. Xie, P. Mäkelä, S.Yashiro, S. Akiyama, and J. M. Davila, Ground level enhancement in the 2014 January 6 solar energetic particle event, ApJL 790:L13 (5pp), 2014.

[2] FAA 1. Extended operations (ETOPS and Polar Operations), Advisory Circ 120-42B, 2008.

[3] FAA 2. In-flight radiation exposure, Advisory Circ 120-61B, 2014.

[4] I. Sandberg, P. Jiggens, D. Heynderickx, and I. A. Daglis, Cross calibration of NOAA GOES solar proton detectors using corrected NASA IMP-8/GME data, Geophys. Res Lett, 11:4435-4441, 8 JUL 2014.

[5] ] S. Roesler and M. Silari, Radioactivity and radiation protection, C34 in J. Beringer et al. (Particle Data Group), Phys Rev D 86, 010001 (2012); updated 2013. 
[6] T. Sato, A. Endo, M. Zankl, N. Petoussi-Henss, H. Yasuda, and K. Niita, Fluence-to-dose conversion coefficients for aircrew dosimetry based on the new ICRP recommendations, Prog Nuc Sci and Tech, 1: 134-137, 2011. 